\title{
Changes in Day/Night Activity in the 6-OHDA-Induced Experimental Model of Parkinson's Disease: Exploring Prodromal Biomarkers
}

\author{
Catalina Requejo ${ }^{1,2 *}$, Karmele López-de-Ipiña ${ }^{3,4}$, José Ángel Ruiz-Ortega ${ }^{5,6}$, \\ Elsa Fernández ${ }^{3}$, Pilar M. Calvo ${ }^{3}$, Teresa Morera-Herreras ${ }^{5,6}$, Cristina Miguelez $^{5,6}$, \\ Laura Cardona-Grifoll ${ }^{1}$, Hodei Cepeda ${ }^{1}$, Luisa Ugedo ${ }^{5,6}$ and José Vicente Lafuente ${ }^{1}$ \\ ${ }^{1}$ LaNCE, Department of Neuroscience, University of the Basque Country (UPV/EHU), Leioa, Spain, ${ }^{2}$ Department \\ of Neurology, Icahn School of Medicine at Mount Sinai, The Friedman Brain Institute, New York, NY, United States, ${ }^{3}$ EleKin \\ Research Group, Department of Systems Engineering and Automation, University of the Basque Country (UPV/EHU), \\ Donostia, Spain, ${ }^{4}$ Department of Psychiatry, University of Cambridge, Cambridge, United Kingdom, ${ }^{5}$ Department \\ of Pharmacology, University of the Basque Country (UPV/EHU), Leioa, Spain, ${ }^{6}$ Autonomic and Movement Disorders Unit, \\ Neurodegenerative diseases, Biocruces Health Research Institute, Barakaldo, Spain
}

\section{OPEN ACCESS}

Edited by:

Martin Witt,

Centre of Transdisciplinary Neuroscience Rostock, Germany

Reviewed by: Andreas Wree,

University of Rostock, Germany Veronica Alexandra Antipova, Medical University of Graz, Austria

Alexander Hawlitschka, University Hospital Rostock, Germany

*Correspondence: Catalina Requejo catalina.requejo@ehu.eus

Specialty section: This article was submitted to

Neurodegeneration,

a section of the journal

Frontiers in Neuroscience

Received: 31 July 2020 Accepted: 24 September 2020

Published: 14 October 2020

Citation

Requejo C, López-de-Ipiña K, Ruiz-Ortega JÁ, Fernández E, Calvo PM, Morera-Herreras T, Miguelez C, Cardona-Grifoll L, Cepeda H, Ugedo L and Lafuente JV (2020) Changes in Day/Night Activity in the 6-OHDA-Induced Experimental Model of Parkinson's Disease:

Exploring Prodromal Biomarkers.

Front. Neurosci. 14:590029.

doi: 10.3389/fnins.2020.590029
The search for experimental models mimicking an early stage of Parkinson's disease (PD) before motor manifestations is fundamental in order to explore early signs and get a better prognosis. Interestingly, our previous studies have indicated that 6-hydroxydopamine (6-OHDA) is a suitable model to induce an early degeneration of the nigrostriatal system without any gross motor impairment. Considering our previous findings, we aim to implement a novel system to monitor rats after intrastriatal injection of 6-OHDA to detect and analyze physiological changes underlying prodromal PD. Twenty male Sprague-Dawley rats were unilaterally injected with 6-OHDA $(n=10)$ or saline solution $(n=10)$ into the right striatum and placed in enriched environment cages where the activity was monitored. After 2 weeks, the amphetamine test was performed before the sacrifice. Immunohistochemistry was developed for the morphological evaluation and western blot analysis to assess molecular changes. Home-cage monitoring revealed behavioral changes in response to 6-OHDA administration including significant hyperactivity and hypoactivity during the light and dark phase, respectively, turning out in a change of the circadian timing. A preclinical stage of PD was functionally confirmed with the amphetamine test. Moreover, the loss of tyrosine hydroxylase expression was significantly correlated with the motor results, and 6-OHDA induced early proapoptotic events. Our findings provide evidence for a novel prodromal 6-OHDA model following a customized monitoring system that could give insights to detect non-motor deficits and molecular targets to test neuroprotective/neurorestorative agents.

Keywords: 6-hydroxydopamine, circadian rhythms, home-cage monitoring system, prodromal biomarkers, prodromal Parkinson's disease symptoms, behavior, non-motor deficits, rat

Abbreviations: ADN, Axodendritic network density; AKT, Protein kinase B; AUC, Area Under the Curve; CL, Contralateral; EE, Enriched environment; ERK, Extracellular signal-regulated kinase; GFAP, Glial fibrillary acidic protein; IL, Ipsilateral; NMS, Non-motor symptoms; 6-OHDA, 6-hydroxydopamine; PD, Parkinson's disease; REM, Rapid eye movement; NREM, Non-rapid eye movement; RBD, Rapid eye movement sleep behavior disorder; ROC, Receiver Operating Characteristics; ROS, Reactive oxygen species; SN, substantia nigra; SNr, Substantia nigra reticulate; TH, Tyrosine hydroxylase; TH-ir, Tyrosine hydroxylase-immunoreactivity; Tpm, Turns per minute; VTA, Ventral tegmental area. 


\section{INTRODUCTION}

Parkinson's disease (PD) is a complex neurologic disorder in which not only motor impairments occur, but also other non-motor symptoms (NMS) play a relevant role, including hyposmia, sleep disorders, depression, constipation and cognitive deficit (Schapira and Tolosa, 2010; Mantri et al., 2018; Weintraub et al., 2018). Most of them often appear earlier than the motor symptomatology, during the so-called prodromal stage and worsen following the disease's progression (Schapira et al., 2017). Indeed, NMS may manifest several years prior to the onset of motor symptoms, even up to 20 years before the diagnosis, and the prevalence can vary between the patients (Schapira et al., 2017; Rees et al., 2019). In this context, although there are not specific NMS for PD, the presence and combination of various NMS as well as the correlation with an early dopamine depletion may be useful for early diagnosis (Berg et al., 2013; Schapira et al., 2017). Remarkably, slight motor symptoms $(<40-60 \%$ of dopaminergic neuronal cell loss) have been also associated to the prodromal stage when they do not meet the criteria for the clinical diagnosis of PD (Mahlknecht et al., 2015). Thus, it is essential to establish a correlation between the early manifestations of PD (prodromal stage) with the clinical and pathological stage for an early diagnosis of PD (Mahlknecht et al., 2015; Liepelt-Scarfone et al., 2017; Sherbaf et al., 2018).

Thus, studies about early events of the disease are emerging in order to find some biomarker for an early-premotor diagnosis (Miller and O'Callaghan, 2015). However, the neuroanatomical and etiological background of NMS in PD remain to be elucidated (Schapira et al., 2017). One of the first NMS that is suffered by a high percentage of the patients is the sleep disorder, such as sleep fragmentation, excessive daytime sleepiness or "REM behavior disorder" (RBD), which is a pathology based on increasing muscle tone during the REM (rapid eye movement) sleep (De Lazzari et al., 2018; De Castro Medeiros et al., 2019). Among the mechanisms underlying sleep disturbance, the disruption in the circadian system is attributed as triggering factor (Willison et al., 2013). Indeed, growing evidences support an association between circadian disruption and PD, suggesting that the dopamine depletion may lead to circadian rhythm irregularities including the alteration of the circadian control of the rest/activity rhythms (Tanaka et al., 2012; De Lazzari et al., 2018).

In the search for preclinical models of PD, there is a main challenge for reproducing those functional and pathological changes that could provide the scaffold to find out about the target for the design of neuroprotective therapies before the progression of the disease (Duty and Jenner, 2011; Ko and Bezard, 2017; Lafuente et al., 2017; Iarkov et al., 2020). In this context, the well-known model of the 6-hydroxydopamine (6-OHDA) which is widely used to study motor deficits, can also be useful for identifying non-motor and early motor impairments specially when combined with advanced technological devices (Grandi et al., 2018). In this model, the site of administration and time elapsed after the injection are critical for determining the extent and time course of the lesion (Kirik et al., 2000; Deumens et al., 2002). In our hands, this model is also useful for studying the preclinical phase of $\mathrm{PD}$, as intrastriatal injection of 6-OHDA in adult male rats induced an early degeneration of the nigrostriatal system without any gross motor impairment as well as upregulation of caspase- 3 and downregulation of survival signaling pathways (Requejo et al., 2018a,b).

Therefore, in the present study we aimed to detect prodromal changes in the behavior following an experimental model of PD based on the intrastriatal injection of 6-OHDA with a short time of evolution (2 weeks) in adult male rats housed in monitored enriched environment (EE) cages.

\section{MATERIALS AND METHODS}

\section{Experimental Design and Housing Conditions}

Twenty adult (3-month-old) male Sprague-Dawley rats, weighting $280-300 \mathrm{~g}$ at the time of surgery, were obtained from Harlan Laboratories, S, A, (Barcelona, Spain). Rats were randomly assigned to the following groups: Sham group (saline-lesioned rats, $n=10$ ) as control and 6-OHDA group (6-OHDA-lesioned rats, $n=10$ ). After stereotaxic injection of 6-OHDA or saline solution, the animals were housed for 2 weeks in monitored enriched environment cages (10 animals per cage) consisting of large cage $(790 \mathrm{~mm} \times 460 \mathrm{~mm} \times 640 \mathrm{~mm})$ with two floors, which were connected by a plastic ramp and an external running wheel (Figure 1A and Supplementary Figure S1). A $12 \mathrm{~h}$ light/12 h dark cycle was established with access to food and water ad libitum. The EE cages were developed by our research group and they were designed to host up to 12 rats as well as housing food feeders, water feeding bottles, two semi-closed rooms, a wide-open area, and a ramp to access a runway situated on the upper floor. In addition to this, these EE cages also provide four gates that may be opened to connect to other cages, runways, or exercise wheels. Current EE cages were supplied with an automatic recording system. This novel system consists, basically, of an infrared camera situated above every cage and connected to a raspberry device programmed to collect pictures accurately every $30 \mathrm{~s}$ (Figure 1A). In fact, a Raspberry Pi (Raspberry Pi 2 Model B, Raspberry Pi products, United Kingdom) is a standalone embedded computer that measures the size of a credit card. It has enough power to run a linux operating system and connect it to several external devices and sensors. In this case, it was connected to Pi NoIR Camera V2 (Raspberry Pi products, United Kingdom) that provided a clear image of the cage even at night's dark due to the infrared lighting that was set up throughout the room (Supplementary Figure S1). This in-house customized automatic system continuously recorded a picture every $30 \mathrm{~s}$ and saved it in an SD Card. At the end of the experiment, more than 60 gigabytes in pictures were collected and processed in order to measure changes in the activity (Figure 1B).

Two weeks after the intrastriatal injection the amphetamine test was performed for evaluating motor deficits and with the purpose of confirming that no rat presented more than 5 turns per minute (tpm) in order to get a preclinical model (Supplementary Table S1). This criterium was established due to the fact that it has previously been shown that 


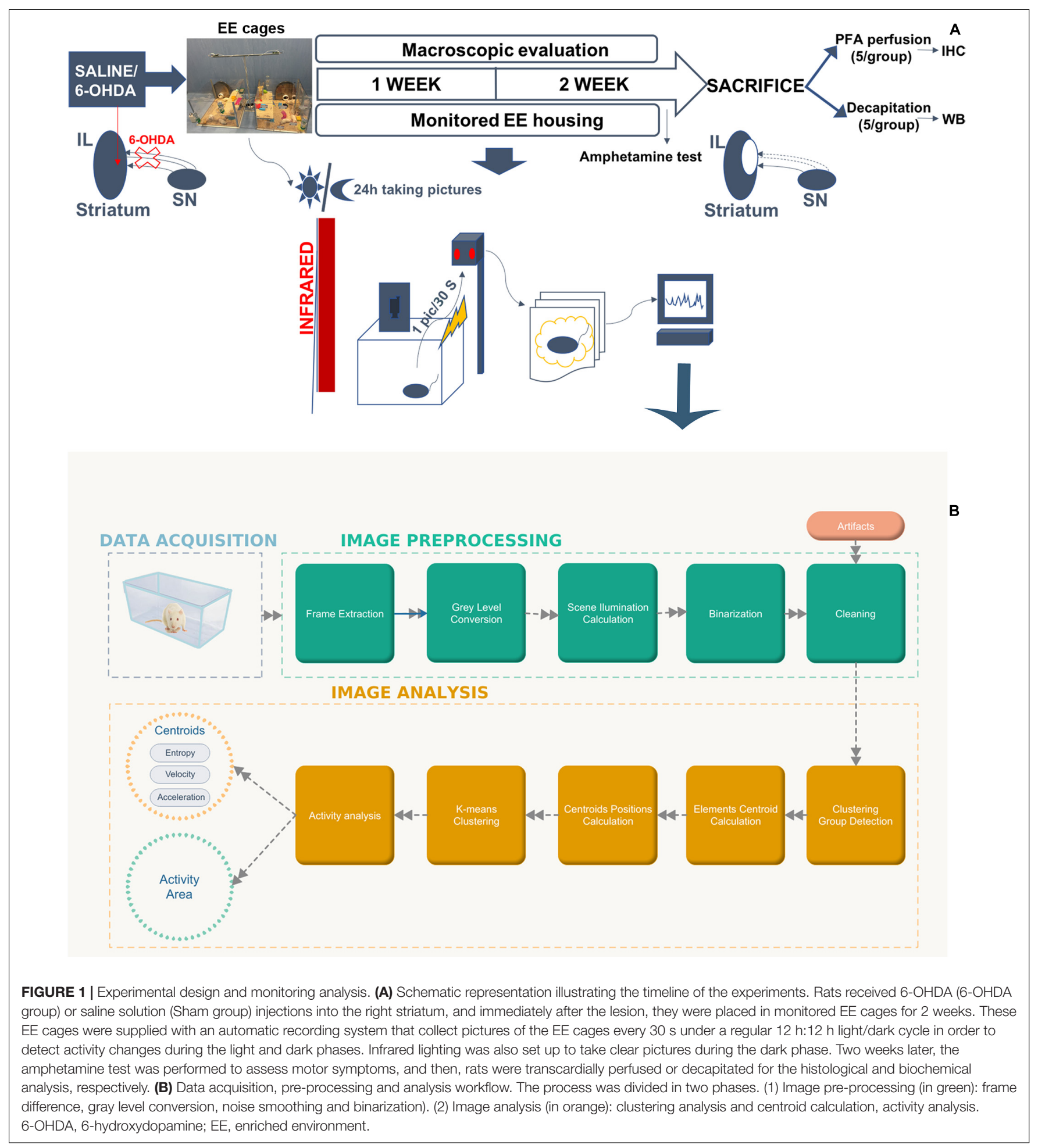

rats rotating more than $5 \mathrm{tpm}$ presented a lesion ranging from 50 to $90 \%$ which may predict motor impairment (Björklund and Dunnett, 2019). After the amphetamine test, rats were sacrificed by transcardial perfusion for the histological evaluation or by decapitation for the biochemical analysis (Figure 1A).
All the experimental protocols were reviewed and approved by the Ethical Committee and Animal Welfare of the University of the Basque Country (UPV/EHU, CEBA M20/2015/024, approval date 12/28/2015), and in accordance with the European Community Council Directive on "The Protection of Animals Used for Scientific Purposes" (2010/63/EU) and 
with Spanish Law (RD 53/2013) for the care and use of laboratory animals.

\section{6-OHDA Lesions}

Rats were lesioned as we previously described (Requejo et al., 2018a,b). Briefly, thirty minutes before surgery, rats were pre-treated with desipramine $(25 \mathrm{mg} / \mathrm{kg}$, i.p., Sigma, St. Louis, United States) and pargyline $(50 \mathrm{mg} / \mathrm{kg}$, i.p., Sigma, St. Louis, United States) in order to preserve the noradrenergic system and the degradation of the toxin, respectively. Then, rats were deeply anaesthetized with isoflurane inhalation (1.5-2\%; Esteve Química, Barcelona, Spain) and placed in the stereotaxic frame (David Kopf ${ }^{\circledR}$ Instruments). 6-OHDA (3 $\mu \mathrm{g} / \mu \mathrm{l}$, in $0.02 \%$ ascorbic acid) or saline solution $(0.9 \% \mathrm{NaCl})$ was infused using a $10 \mu \mathrm{l}$ Hamilton syringe fitted with a 26-gauge needle at a rate of $0.5 \mu \mathrm{l} / \mathrm{min}$ by a single syringe infusion pump (KDS Scientific, MA, United States).

To generate a mild lesion of the nigrostriatal pathway, three injections of $2.5 \mu \mathrm{l}$ of the 6-OHDA solution (a total volume of $7.5 \mu \mathrm{l})$ were administered at three coordinates into the right striatum, relative to the bregma and dura, with the toothbar set at -2.4 : anteroposterior $(\mathrm{AP})+1.3 \mathrm{~mm}$, mediolateral $(\mathrm{ML})+2.8 \mathrm{~mm}$, dorsoventral (DV) $-4.5 \mathrm{~mm}$; AP $-0.2 \mathrm{~mm}$, $\mathrm{ML}+3.0 \mathrm{~mm}, \mathrm{DV}-5.0 \mathrm{~mm}$ and $\mathrm{AP}-0.6 \mathrm{~mm}, \mathrm{ML}+4.0 \mathrm{~mm}$, DV $-5.5 \mathrm{~mm}$, according to Paxinos and Watson atlas (Paxinos and Watson, 2013). The needle was left in place for an additional $5 \mathrm{~min}$ to allow the toxin to diffuse into the structure, and then it was slowly retracted. 6-OHDA was prepared daily for each surgery session and changed every $2-3 \mathrm{~h}$. The 6-OHDA solution was kept on ice and protected from light during the surgery to avoid oxidation, which would be indicated by a color change (clear to brown-pink color). Control rats received the same volume of saline solution in the same manner. In addition, a short time of evolution ( 2 weeks) was elapsed before sacrifice.

\section{Monitoring Analysis}

The in-house customized automatic system based on the image processing with the infrared Camera Module v2 (Pi NoIR; Raspberry Pi Products, United Kingdom) was used for the analysis of the rat group behavior. A schematic diagram of the working procedure for image processing is described in Figure 1B.

This process was divided in two phases: image pre-processing and image analysis.

1. In the first phase video sequences were acquired by a highquality camera and pre-processed by an own toolbox in MATLAB to create series of binary image frames of the rat group activity. The Toolbox includes: (1) function for image processing, (2) Activity analysis (centroid analysis), and (3) Period analysis among others. Thus, the activity areas (motion detection) were calculated based on a frame difference method (Manchanda and Sharma, 2016; Sengar and Mukhopadhyay, 2016). Frame absolute difference was calculated between two consecutive frames with a sampling period of $30 \mathrm{~s}$. Images were converted from Red Green Blue
(RGB) which is a standard format for color image, into gray scale, after that in binary images and then by a threshold filter extracted the main activity area of the system (rats, and objects moved by the rats). Finally, activity areas were defined applying morphological filters to reduce the noise and for smoothing the images. In particular, morphological filters were applied using MATLAB function bwmorph:

Bwmorph (Ir'open');\% Remove peaks

Bwmorph (Ir'close');\% Remove hole

2. During the second phase the trajectories of the animal group is generated by the system centroid (the average position of all the activity areas in the binary image) evolution. The centroid is estimated by $k$-means algorithm over the binarized frame series (Eguiraun et al., 2018; López-de-Ipiña et al., 2019). Thus, within each frame, the coordinates of the centers of every object were calculated, and K-means were applied to find the center of the entire group, that is the "centroid." A centroid with two coordinates:

$$
C=\left(x_{c}, y_{c}\right)
$$

3. The group trajectory consists of the evolution of that centroid from the first frame to the last one.

Finally, the rat groups' behavior was described and modeled by the following parameters:

1. Number of activity areas in the binary images.

2. Shannon entropy of the centroid trajectory. Shannon entropy is a main concept in information theory and is a measure of average uncertainty (information content). Entropy in biosignals gives information about the system evolution and behavior and can be applied to analyze pathological behaviors (Eguiraun et al., 2018; López-deIpiña et al., 2019).

Velocity, speed and acceleration defined as:

$$
\left\{\begin{array}{l}
v_{c x}=\Delta x_{c} / \Delta t \\
v_{c y}=\Delta y_{c} / \Delta t
\end{array}\right.
$$

being speed the absolute value of velocity. An acceleration:

$$
\left\{\begin{array}{l}
a_{c x}=\Delta v_{c x} / \Delta t \\
a_{c y}=\Delta v_{c y} / \Delta t
\end{array}\right.
$$

\section{Amphetamine-Induced Rotation Test}

Two weeks after intrastriatal injection the amphetamine test was developed following the previously described methodology for this behavioral test (Miguelez et al., 2011). Briefly, D-amphetamine $(5 \mathrm{mg} / \mathrm{kg}$ in $0.9 \% \mathrm{NaCl}$; Sigma-Aldrich, St. Louis, United States) was intraperitoneally administered and the animals were placed in an individual circular cage (rotameter). In the present study, we used a higher dose of D-amphetamine in order to get rotational behavior due to the lesion was minor (Björklund and Dunnett, 2019). After $15 \mathrm{~min}$ of latency, the total number of full ipsilateral (IL) rotations was recorded during $90 \mathrm{~min}$ (Multicounter LE3806; Harvad 
Apparatus, Holliston, MA, United States) in order to include the entire response period and avoid differences in variability due to the differences in the pharmacokinetics or in the dopamine release kinetics between rats (Miguelez et al., 2011; Björklund and Dunnett, 2019). Data were expressed as the number of turns per minute.

\section{Morphological Analysis}

Morphological evaluation was performed as we previously described (Requejo et al., 2015, 2018a,b).

\section{Tissue Processing for Histological Evaluation}

After behavioral test, five rats from each group were intraperitoneally anesthetized with chloral hydrate at $20 \%$ (Ref: 141,975, Panreac Quimica SA, Barcelona, Spain) and transcardially perfused with $0.9 \%$ sodium chloride followed by $4 \%$ paraformaldehyde (PFA) in $0.1 \mathrm{M}$ phosphate-buffered saline (PBS) pH 7.4. Brains were removed, post-fixed overnight in the same fixative solution and next transferred into a cryoprotective solution containing $30 \%$ sucrose and $0.1 \mathrm{M}$ PBS pH $7.4 .50 \mu \mathrm{m}$ serial coronal sections containing striatum and substantia nigra (SN) were obtained with a freezing microtome and collected following the Paxinos and Watson atlas (Paxinos and Watson, 2013 ) in $0.6 \%$ sodium azide in $0.1 \mathrm{M} \mathrm{PBS} \mathrm{pH} 7.4$ for storage and further analysis.

\section{TH Immunostaining}

Tyrosine hydroxylase (TH) immunohistochemical staining was developed on free-floating coronal slices. Briefly, sections were treated with $3 \% \mathrm{H}_{2} \mathrm{O}_{2}$ and $10 \%$ methanol in potassium phosphate buffered saline (KPBS), preincubated with 5\% normal goat serum (NGS) and $1 \%$ Triton X-100 in KPBS (KPBS-T) for $1 \mathrm{~h}$ at room temperature (RT), and later incubated overnight at $4^{\circ} \mathrm{C}$ with rabbit polyclonal anti-TH (Ref: AB-152, Millipore; 1:1,000) in $\mathrm{KPBS} / \mathrm{T}$ containing 5\% NGS, followed by incubation with a secondary biotinylated goat anti-rabbit IgG antibody (Ref: BA1000, Vector Laboratories, Burlingame, CA; $1: 200$ ) in 2.5\% NGS KPBS/T for $2 \mathrm{~h}$. Afterward, all sections were processed with the avidin-biotin-peroxidase complex for $1 \mathrm{~h}$ using a commercial kit (Ref. PK-6102, Elite ABC kit, Vector Laboratories, Burlingame, CA) and the reaction was shown by using 3,3diaminobenzidine (DAB).

Images were visualized, captured and analyzed at $4 \mathrm{x}$ and $40 \mathrm{x}$ magnification by an Olympus BX-50 photomicroscope.

\section{Double Immunofluorescence Staining of Caspase-3 and NeuN}

Coronal sections were incubated with rabbit anti-caspase 3 (H-277) (Ref: sc-7148, Santa Cruz Biotechnology Inc., Spain; 1:50) and monoclonal mouse anti-NeuN (Ref: MAB377, Chemicon International, Inc., Spain; 1:100) diluted in 5\% bovine serum albumin (BSA) and $0.1 \%$ Triton $\mathrm{X}-100$ in PBS overnight at $4^{\circ} \mathrm{C}$. Sections were subsequently incubated with secondary antibodies conjugated to Alexa Fluor-488 (Ref: A11029; Invitrogen; 1:400) and Alexa Fluor-568 (Ref: A11036 Invitrogen; 1:400) for $1 \mathrm{~h}$ at room temperature in darkness. Immunostained sections were further reincubated with
Hoechst for nuclear counterstaining for $10 \mathrm{~min}$, then slices were washed, mounted on glass slides and coverslipped using Vectashield mounting medium for fluorescence (Ref: x-0517; Vector laboratories).

Images were finally analyzed at $20 \mathrm{x}$ magnification using Olympus Fluoview FV500 confocal microscope.

\section{Stereological Analysis}

The unbiased stereological analysis was performed as previously described (Requejo et al., 2015, 2017, 2018a,b) by using a computerized image analysis system (Mercator Image Analysis system, Explora Nova, La Rochelle, France) connected to an Olympus BX-50 photomicroscope. For this purpose, a total of 7-8 sections in a 1: 8 series were analyzed to cover the entire striatum and $\mathrm{SN}$ for each animal.

In brief, in order to evaluate the $\mathrm{TH}$-immunoreactivity (ir) in the IL striatum, the volume of the preserved striatum was calculated using the Cavalieri method (Howard and Reed, 2004) available on Mercator image analysis system by delimiting the negative areas and the entire striatum at $4 \mathrm{x}$ magnification, and multiplying these measurements by the thickness of the slices and the intersectional distance (Requejo et al., 2015, 2018a,b). Values were expressed as the percentage of TH-ir volume of the IL striatum respect to the total volume of the IL one.

Changes in the density of dopaminergic neurons and axodendritic network (ADN) were evaluated in both hemispheres through quantifying the TH-ir neuronal density in the entire SN and the TH-ir ADN density in the $\mathrm{SN}$ reticulata $(\mathrm{SNr})$ using a stereological tool (an optical fractionator) provided by the Mercator image analysis system (West et al., 1991). Once the region of interest was outlined at $4 \mathrm{x}$ magnification, probes of $50 \times 50 \mu \mathrm{m}$ separated by $100 \mu \mathrm{m}$ were launched into the SN. THir neurons and $\mathrm{ADN}$ inside the probe, or crossing the right side of the $\mathrm{X}-\mathrm{Y}$ axis, were counted at $40 \mathrm{x}$ magnification in the entire $\mathrm{SN}$ and $\mathrm{SNr}$, respectively. Data were expressed as the percentage of TH-ir neurons or ADN presents on the IL side (lesioned side) vs. the contralateral (CL) hemisphere (non-lesioned hemisphere).

The study of the topological distribution was performed following the previously described approach (Requejo et al., 2015, 2017). For each animal, sections containing the striatum and the SN were evaluated at three representative rostro-caudal levels, respectively, according to Paxinos and Watson's Atlas (Paxinos and Watson, 2013): rostral (bregma $+0.70 \mathrm{~mm}$ ), middle (bregma, $-0.26 \mathrm{~mm}$ ), and caudal (bregma, $-0.80 \mathrm{~mm}$ ) striatal sections were considered to determine the TH-ir volume; and rostral (bregma, $-5.20 \mathrm{~mm}$ ), middle (bregma, -5.60 ), and caudal (bregma, -6.04) nigral sections were examined to estimate the TH-ir neuronal and ADN density.

\section{Biochemical Analysis Western Blotting}

After the behavioral test, 5 rats of each group were anesthetized with chloral hydrate at 20\% (Ref: 141,975, Panreac Quimica SA, Barcelona, Spain) and decapitated with a rodent guillotine to obtain fresh brain tissue. IL and CL striatum and SN were collected by microdissection and quickly frozen (Chiu et al., 2007). For this purpose, once brains were removed, 
coronal slices containing the striatum and $\mathrm{SN}$ were cut out and subjected to biopsy with a small biopsy needle. Firstly, the cerebellum was separated, and the brain was cut bi-half into the right and left hemisphere, next cut was for removing the olfactory bulb at the level of the optic chiasm, and the fourth cut was at the level of the pituitary stalk, separating the two coronal slices, one of them containing the striatum and the other one the SN. The frontal cortex from the slice containing striatum was removed in the fifth cut, obtaining the striatum. The SN was collected in the sixth, separating the cortex from the slice. Samples were stored at $-80^{\circ} \mathrm{C}$ until the analysis.

Brain slices were manually homogenized $(1: 20 \mathrm{w} / \mathrm{v})$ in lysis buffer [10 mM phosphate-buffered (PB) $(\mathrm{pH} 7.4), 5 \mathrm{mM}$ ethylenediaminetetraacetic acid (EDTA), $5 \mathrm{mM}$ ethyleneglycolbis(2-aminoethylether)-tetraacetic acid (EGTA), $1 \mathrm{mM}$ dithiotreitol (DTT)] containing a protease inhibitor cocktail (Ref: P-8340, Sigma-Aldrich, Spain). Samples were centrifuged $\left(13,000 \mathrm{rpm}\right.$ at $4^{\circ} \mathrm{C}$ for $\left.15 \mathrm{~min}\right)$ and soluble proteins were recovered in the supernatants and quantified using the Bio-Rad Protein Assay (Ref: 500-0006, Bio-Rad Laboratories SA, Spain) based on Bradford's method (Bradford, 1976).

As previously described (Requejo et al., 2018a,b), for each sample $20 \mu \mathrm{g}$ of proteins was loaded into polyacrylamide CRITERION TGX 12\% gels (Bio-Rad Laboratories Inc., Spain) for electrophoresis and then transferred to a PVDF membrane in a Trans-Blot Turbo Transfer System (Bio-Rad, United States) for $7 \mathrm{~min}$. Membranes were incubated with the following primary antibodies: rabbit anti-Phospho-protein kinase B (AKT) (Ser 473) (1:1,000), rabbit anti-AKT $(1: 1,000)$, rabbit anti-Phosphop44/42 MAPK (extracellular signal-regulated protein kinases 1 and 2 (ERK 1/2)] (Thr202/204) (1:1,000), rabbit anti-P44/42 MAPK (ERK 1/2) (1:1,000) (all of them from Cell Signaling Technology Inc., United States), rabbit anti-caspase 3 (H-277) $(1: 1,000)$ (Santa Cruz Biotechnology Inc., Spain), rabbit anti$\beta$-Actin $(1: 2,000)$ (Sigma-Aldrich, Spain) and rabbit anti-BetaTubulin $(1: 1,000)$ (Novus Biologicals, United States) at $4^{\circ} \mathrm{C}$ overnight. Afterward they were incubated with anti-rabbit IgG peroxidase conjugated secondary antibodies (1:2,000) (SigmaAldrich, Spain) for $2 \mathrm{~h}$ at RT and immunoblots were developed with an enhanced chemiluminescence kit (GE Healthcare Life Science, United Kingdom). The luminescence of the reaction product was detected in a personal scanner, LI-COR C-DiGit (LICOR, Bonsai Advanced Technologies SL, Spain), and visualized bands were analyzed with Image Studio Lite 4.0 software (LICOR, Bonsai Advanced Technologies SL, Spain). $\beta$-Actin and $\beta$-tubulin were used as loading controls.

\section{Statistical Analysis}

All results were expressed as the mean \pm SEM (standard error mean). Statistical analysis was developed with GraphPad Prism (v 5; GraphPad Software, Inc., United States) and SPSS Statistics (v 20; IBM Corporation, Armonk, NY, United States). Prior to the analysis, the Shapiro-Wilk test was used to assess the normal distribution of the samples, and Levene's test was used to determine the homogeneity of variance. Mann-Whitney $U$-test was performed to assess differences between groups and within groups in the monitoring analysis. The ROC (Receiver
Operating Characteristics) curve was performed and the AUC (Area Under the Curve) was calculated in order to measure the accuracy of the model which are a metrics for checking classification models (Hanley and McNeil, 1982; Fawcett, 2006), in this case: Sham group and 6-OHDA group. The behavioral data, stereology and densitometry results were analyzed by means of the two-tailed unpaired Student's $t$-test to compare differences between groups. A one-way analysis of variance (ANOVA) followed by Tukey's multiple-comparisons test was used to test the differences between rostro-caudal gradients within each experimental group. The correlation was examined by Pearson product-moment correlation coefficient. Values $P<0.05$ were considered statistically significant.

\section{RESULTS}

\section{Behavioral Evaluation}

The monitoring of EE cages allows obtaining data about activity and centroid features (speed, acceleration and entropy) during the light/dark cycle. Remarkably, 6-OHDA-lesioned rats decreased significantly the number of activity areas during the dark phase, which is the active period of the rodent circadian cycle, comparing to Sham group (*** $p<0.0001$, Mann-Whitney $U$-test) (Figure 2B). Moreover, the 6-OHDA group also showed a significant hyperactivity during the light phase (resting period) in comparison with control rats which may be related to a sleep behavior disruption $\left({ }^{* * *} p<0.0001\right.$, Mann-Whitney $U$-test; Figures 2A,B). Overall, these results suggested that animals from the 6-OHDA group switched their transitional rhythms respect to the control group. Furthermore, the effect of 6-OHDA on the disruption of circadian rhythms was also supported by a significant difference in the activity between both light and dark phases which was also seen in Sham group $\# \# \# p<0.0001$ activity area mean in light cycle vs. activity area mean in dark phase within the same group, Mann-Whitney $U$-test; Figure 2B).

In line with these results, although Sham group showed more remarkable changes between dark and light cycles in terms of speed and acceleration than 6-OHDA group, entropy was significantly higher in the 6-OHDA group (6-OHDA group vs. Sham group, ${ }^{* * *} p<0.0001$, Mann-Whitney $U$-test; Figures 2C-E). The fact that entropy was higher supported and confirmed the incipient sleep pathological condition (Figure 2E). Both groups also showed significant differences between both dark and light phases in all the examined parameters related to the centroid evolution indicating different behaviors in both

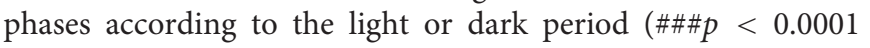
within the same group, Mann-Whitney $U$-test; Figures 2C-E). Besides, the 6-OHDA effect on the acceleration and speed in both phases pointed to a difficulty in the movement noticed in both phases.

Thus, this is a model that allows integrating information about both dark and light phases.

Accordingly, the efficiency of this model was confirmed by the results obtained by the ROC curve and its AUC which measure the accuracy of the model by Logistic Regression. In the ROC curve the true positive rate (Sensitivity) is represented in function 

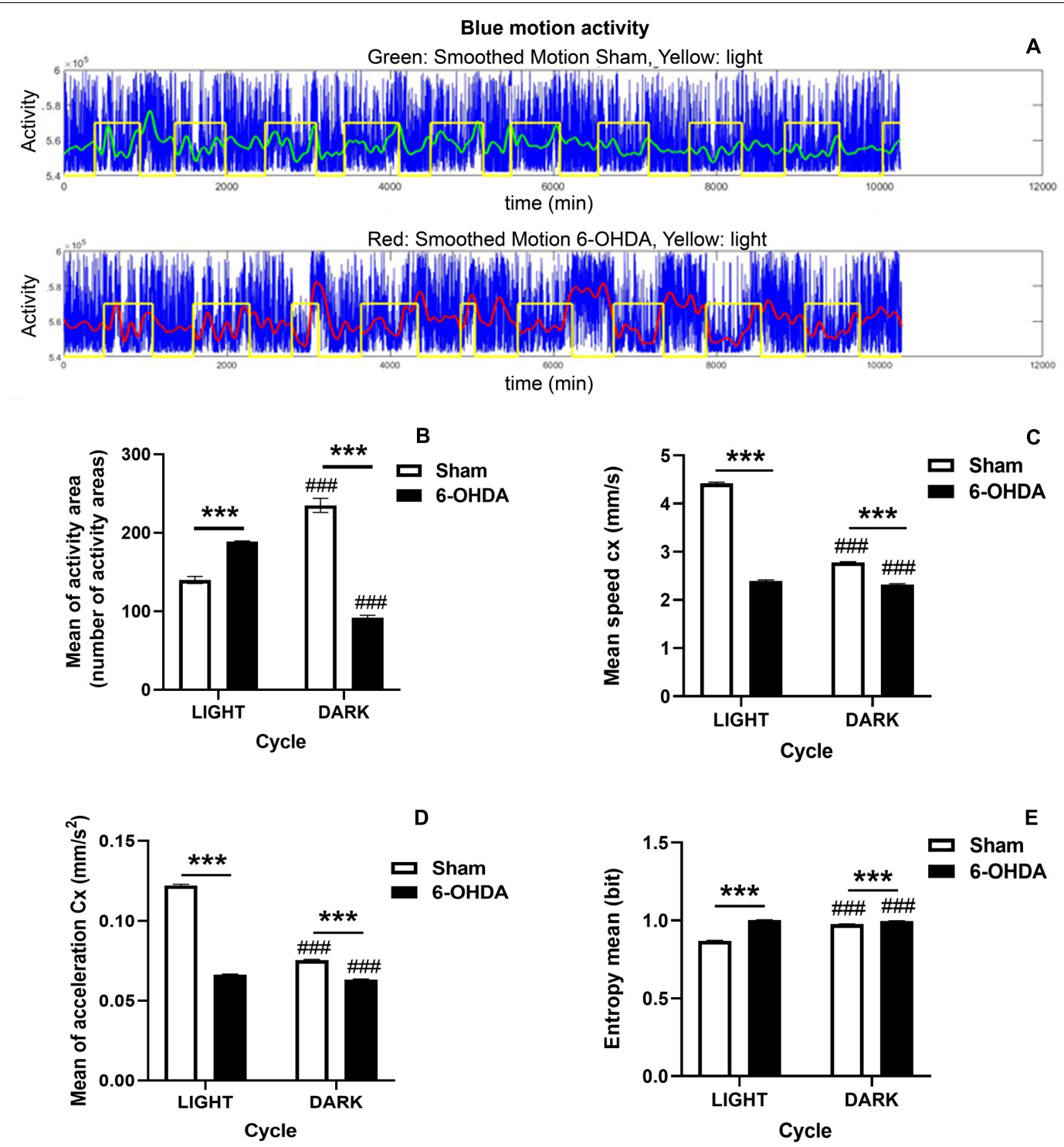

FIGURE 2 | Circadian rhythm disruption in 6-OHDA-induced rats. (A) Diagram illustrating the motion activity signal in 6-OHDA (upper panel) and Sham (bottom panel) groups during the whole manuscript. Afterward, the data are preprocessed and selected in order to obtain for the statistical analysis, appropriated balanced datasets of the light and dark periods for the two groups. Blue: motion activity signal, Green: smoothed signal for control group, Red: smoothed signal for 6-OHDA group, Yellow: light level. Graphs depict significant activity changes (B), the centroid features including the mean of centroid speed (C), the mean of centroid acceleration (D) and the entropy mean (E) between 6-OHDA and Sham groups during the light/dark cycle and within the same group. Data are expressed as mean \pm SEM. General analysis was performed by considering $12 \mathrm{~h}$ of dark/light periods. Statistical differences appear $(p<0.05)$ for Mann-Whitney $U$-test between both groups and within the same group for both light and dark phases (6-OHDA group vs. Sham group, ${ }^{\star \star *} p<0.001$; and light cycle vs. dark cycle within the same group, $\left.{ }^{\# \#} p<0.001\right)$.

of the false positive rate (1-Specificity) for different operating points, features of the system, where an AUC of $100 \%$ would represent the highest specificity and sensibility of the model to separate both groups. Our data gave a ROC curve (each point on the curve represents a sensitivity/specificity) that reached a significant AUC of 0.854 giving $85 \%$ of sensibility and specificity to this model ( $0.854 \pm 0.0047, p=0$; Figure 3).

On the other hand, minimal motor deficits were observed in the amphetamine test (Supplementary Table S1). Although animals increased significantly the number of IL turns per minute (tpm) respect to control group, $(1.93 \pm 0.39$ tpm vs. $0.32 \pm 0.076$ tpm; $t_{(17)}=4.292, p=0.0005$, Unpaired Student's $t$-test), no rats turned more than 3.98 turns per minute $(0.79-3.98$ tpm; Supplementary Figure S2).

Therefore, a prodromal stage would be supported by the mild presence of motor symptoms.

\section{Morphological Evaluation}

Once confirmed the functional changes displayed by 6-OHDA group 2 weeks after 6-OHDA administration, we also assessed 


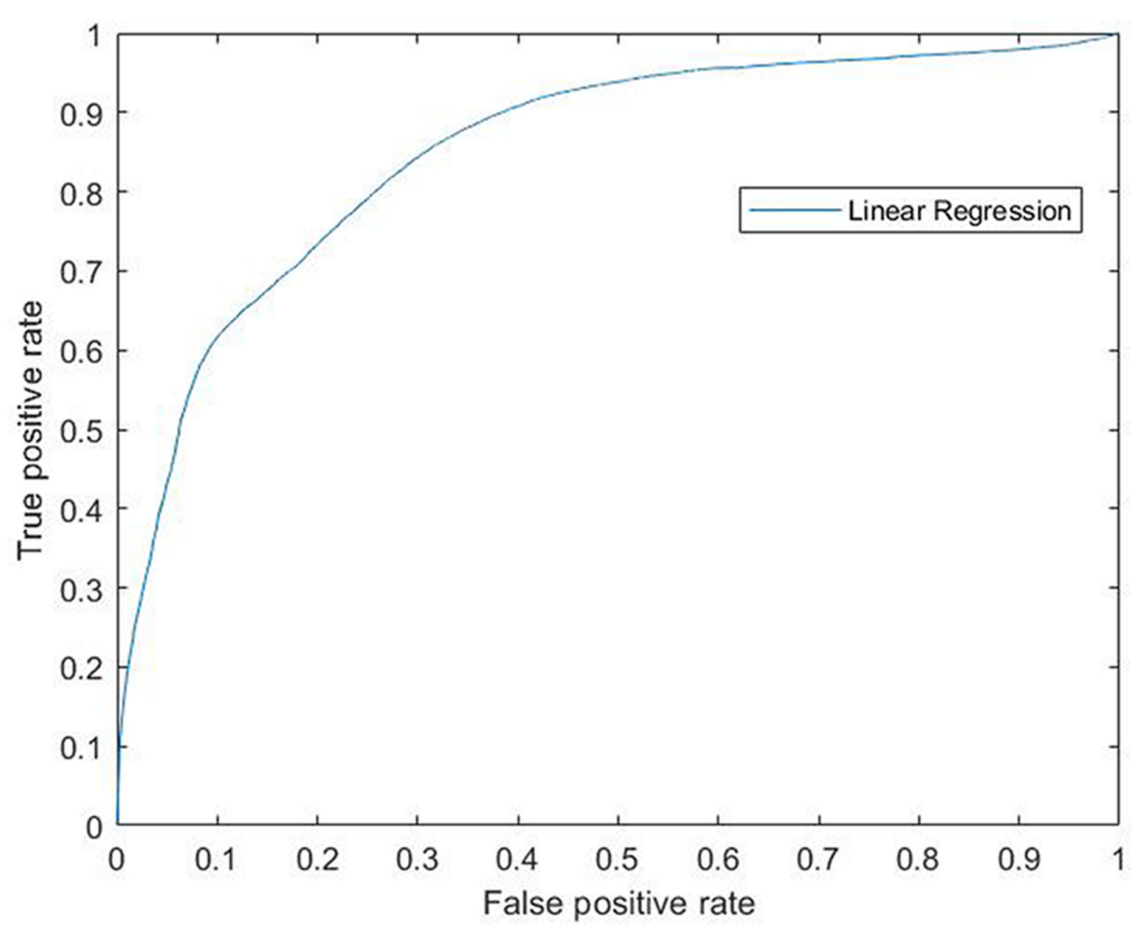

FIGURE 3 | ROC curve for linear regression. Modeling for 6-OHDA and Sham groups. ROC curve (each point on the curve represents a sensitivity/specificity) that reached a significant AUC of 0.854 giving $85 \%$ of sensibility and specificity to this model $(0.854 \pm 0.0047, p=0)$. ROC, Receiver Operating Characteristics; 6-OHDA, 6-hydroxydopamine; AUC, Area Under the ROC Curve.

whether 6-OHDA exerted a histological effect that we had previously observed 3 weeks after 6-OHDA administration (Requejo et al., 2018a,b).

\section{6-OHDA-Induced Mild Nigrostriatal Dopaminergic Degeneration After a Short Evolution Time}

Analysis of $\mathrm{TH}$-immunostained sections from the striatum and $\mathrm{SN}$ confirmed that, at this survival time, 6-OHDA induced a moderate $\mathrm{TH}$-ir fiber loss either in the striatum or in the $\mathrm{SNr}$ (Figure 4). In addition, dopaminergic neurons in the $\mathrm{SN}$ were not notably reduced (Figures $4 \mathrm{E}, \mathrm{G}$ ). Rostro-caudal study of $\mathrm{TH}$-ir in the $\mathrm{SN}$ indicated that the number of the survival TH-ir neurons as well as the TH-ir axodendritic network (ADN) in 6-OHDA group increased rostro-caudally. Interestingly, one-way ANOVA followed by Turkey post hoc test indicated significant differences $\left({ }^{*} p<0.05\right)$ between rostral and caudal levels within the 6-OHDA group regarding the number of $\mathrm{TH}$ ir neurons $(35.61 \pm 8.89 \%$ of $\mathrm{TH}$-ir neuronal cells at the rostral sections vs. $70.12 \pm 7.03 \%$ of $\mathrm{TH}$-ir neuronal cells at the caudal sections; $p=0.031$ ). However, regarding the number of TH-ir AND no significant differences were found between rostral and caudal sections [52.24 $\pm 6.32 \%$ of $\mathrm{TH}$-ir $\mathrm{ADN}$ at the rostral sections vs. $76.86 \pm 5.29 \%$ of TH-ir of $\mathrm{ADN}$ at the caudal section; One-way ANOVA, $F_{(2,9)}=3.800, p=0.063$; Figures $\left.4 \mathrm{G}, \mathbf{H}\right]$. In addition to this, no topological distribution in the $\mathrm{TH}$-ir striatal fibers was appreciated (Figure 4D).

\section{Correlations Between Motor and Morphological Changes}

The number of IL amphetamine-induced rotations was strongly correlated with the percentage of $\mathrm{TH}$-ir neuronal cell loss $(r=-0.94)$ and it was statistically significant $(p \leq 0.05)$ (Figure 5B). On the other hand, the TH-ir terminal loss either in striatum or in $\mathrm{SN}$ also showed a correlation with the increase in the number of IL rotations per minute close to be statistically significant $(r=-0.84, p=0.073$ for TH-ir IL striatal volume and $r=-0.93, p=0.065$ for TH-ir AND; Figures 5A,C).

\section{Biochemical Analysis}

In order to assess changes in apoptosis, the expression of caspase3 in neuronal cells was morphologically evaluated by double immunostaining against NeuN (nuclear marker of neuronal cells) and caspase-3 (apoptosis marker). In addition, caspase-3 levels were also biochemically analyzed by western blot in the striatum and SN (Figure 6).

Double immunofluorescence revealed higher caspase-3 immunoreactivity and lower NeuN immunoreactivity in 6-OHDA-lesioned rats compared to Sham group in the IL striatum as well as in the IL SN. In fact, 6-OHDA induced a remarkable apoptosis in neuronal cells due to a considerable amount of NeuN positive cells co-localized with caspase-3 in 6-OHDA group (Figures 6A,C).

Furthermore, immunoblot results were consistent with the outcomes showed by immunostaining. The expression of 

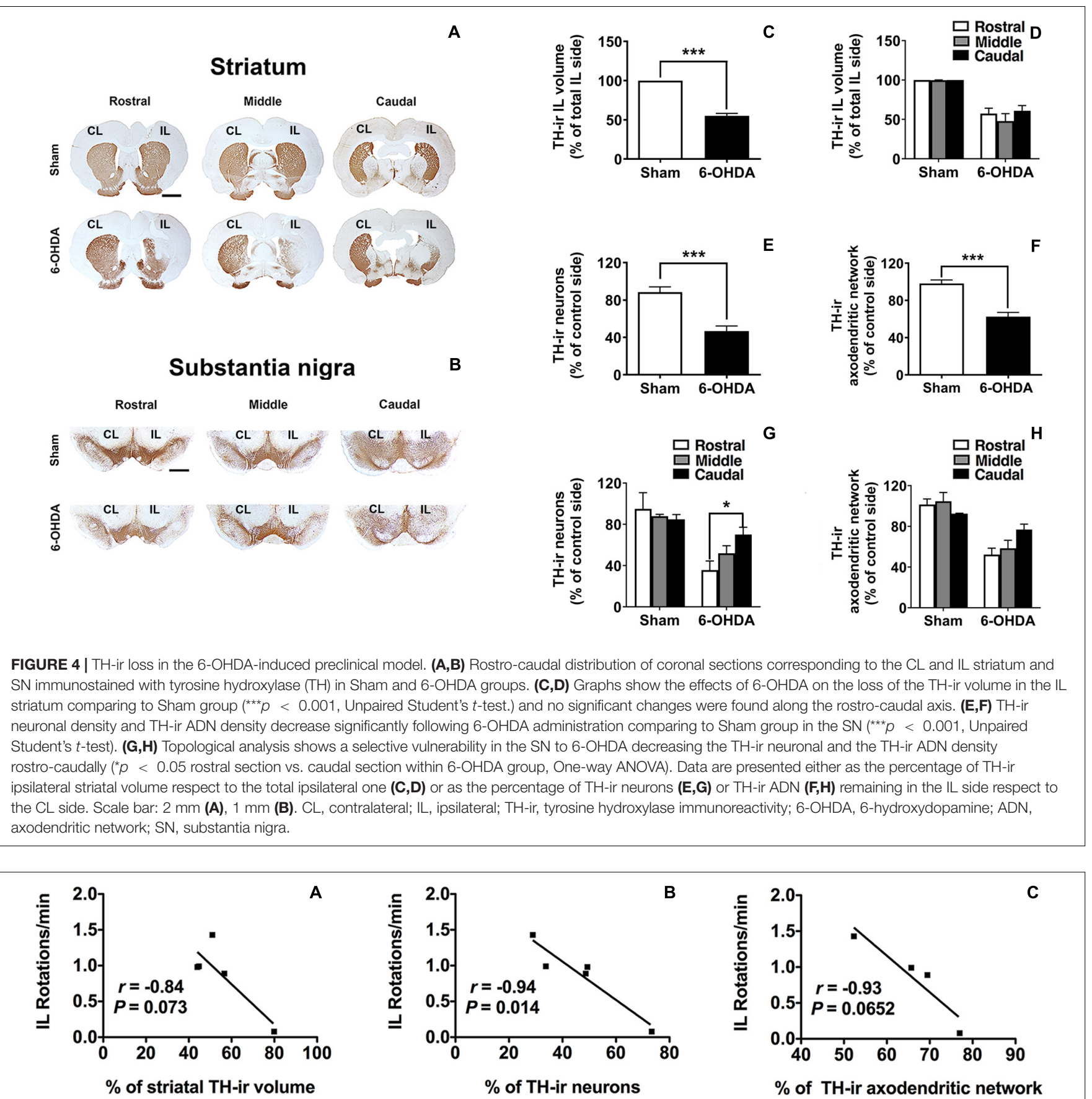

FIGURE 5 | Morpho-functional correlation between amphetamine-induced rotations and dopaminergic neurons in SN in addition to dopaminergic terminals in both striatum and SN after 6-OHDA administration. (A) Amphetamine-induced IL rotations correlate negatively with the \% of TH-ir striatal volume IL, (B) also with the \% of TH-ir neuronal density and (C) with the \% of TH-ir ADN density. Significance. $p<0.05$, IL rotations vs. TH-ir neurons. SN, substantia nigra; 6-OHDA,

6-hydroxydopamine; TH-ir, tyrosine hydroxylase immunoreactivity; IL, ipsilateral.

caspase-3 was upregulated in 6-OHDA-lesioned rats in the striatum and in SN following a similar pattern $(127.9 \pm 5.1 \%$ and $127.9 \pm 26.08 \%$, respectively), while caspase- 3 activation was barely evidenced in Sham group $(103.8 \pm 7.6 \%$ in the striatum and $90.7 \pm 7.57 \%$ in the $\mathrm{SN}$ ). However, statistically significant differences between both groups were only found in the striatum $\left[t_{(7)}=2.426, p=0.045\right.$, Unpaired Student's $t$-test; Figures 6B,D $]$.

However, no significant changes were seen in the prosurvival signaling protein levels (AKT and ERK) between 6-OHDA and Sham groups 2 weeks postinjection, despite 


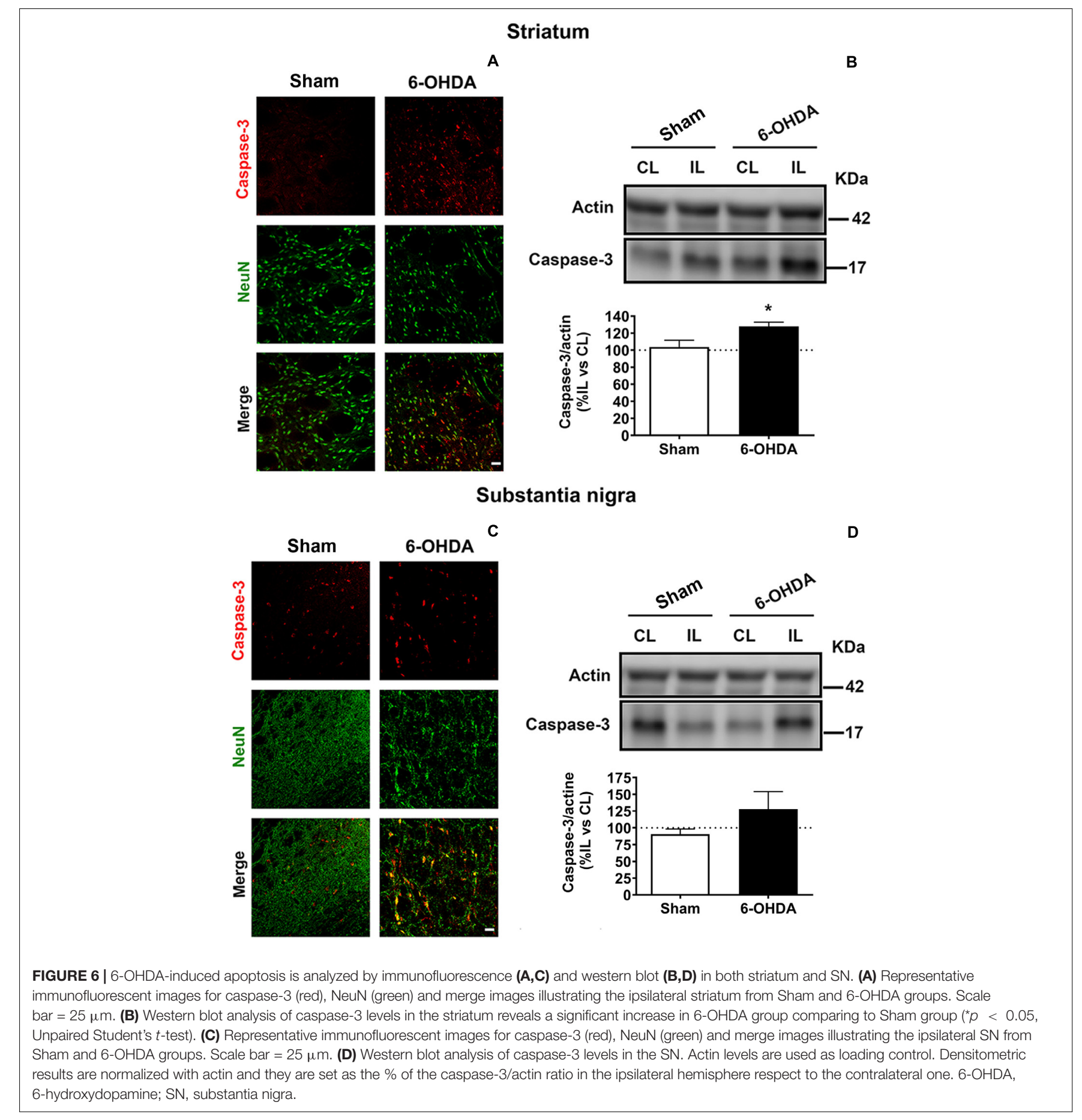

both proteins were downregulated in the 6-OHDA group (Supplementary Figure S3).

\section{DISCUSSION}

In the present study, we achieved to monitor the alteration of the circadian cycle in a preclinical model with an automatized and non-invasive method. Indeed, our results evidenced that 2 weeks after the administration of 6-OHDA in the striatum of adult male rats despite inducing only a subclinical motor deficit, other physiological impairments associated with the premotor phase of $\mathrm{PD}$ were found. These were related to mild morphological and molecular changes in the dopaminergic system.

Remarkably, our results suggested that 6-OHDA-lesioned rats showed sleep behavioral disruption as an effect of dysfunctional circadian clock. Circadian disruption consists 
in the alteration at the level of the daily rest/activity rhythm (De Lazzari et al., 2018) and involves to multiple systems, thereby some authors speculate that circadian disfunction can worsen the progression of PD (Willison et al., 2013; Fifel, 2017; De Castro Medeiros et al., 2019). Thus, the use of PD models that reproduce sleep and circadian abnormalities as well as the design of systems that aid to collect data about the evolution of the activity changes in circadian rhythms as we described in this study represent a good strategy for predicting PD prior to the clinical motor deficits manifestation.

In particular, our findings indicated that 6-OHDA-lesioned rats switched their circadian rhythms respect to the control animals reflecting alterations in the temporal patterning of sleep as was previously described in PD patients (Willison et al., 2013). Thus, we can speculate that this change in the pattern of rest/activity seen in response to 6-OHDA may be similar to the insomnia at night or the hypersomnia during the daytime experimented by PD patients (De Castro Medeiros et al., 2019). Behavioral changes related to circadian cycles were already described in the 6-OHDA model, such as alterations on circadian locomotor rhythm parameters (Ben and Bruguerolle, 2000; Gravotta et al., 2011; Masini et al., 2017; De Lazzari et al., 2018; Souza et al., 2018; Ciric et al., 2019; De Castro Medeiros et al., 2019). In fact, some of these studies associated the circadian disruption with the loss of dopaminergic neurons indicating that dopamine may act as a modulator of the circadian system (Isobe and Nishino, 2001; Gravotta et al., 2011; De Lazzari et al., 2018; Souza et al., 2018; Ciric et al., 2019; De Castro Medeiros et al., 2019). Interestingly, our findings are in agreement with these studies, however, we detect changes in both light and dark phases on a regular 12:12 light-dark cycle during 2 weeks after unilateral striatal 6OHDA lesion. Thus, we also suggest that the early alteration on the circadian rhythms may help to predict the dopamine depletion. In line with our results, Sakata and collaborators found alterations in the sleep behavior in both, light and dark phases. They reported after bilateral injection of 6-OHDA into the ventral tegmental area (VTA) a decrease in REM sleep during the light phase contrary to the dark phase, in which they observed an increase in REM sleep and in non-rapid eye movement (NREM) as well as a reduction in spontaneous activity (Sakata et al., 2002).

The tendency to decrease the activity during the dark phase in response to 6-OHDA was also in accordance with other studies, some of them suggested that 6-OHDAlesioned rats may show slightly motor impairment and more diurnal activity during the first week after 6-OHDA administration into the SN (Souza et al., 2018). While other studies did not detect activity change during the light phase (Masini et al., 2017).

In addition, 6-OHDA-induced activity changes were also supported by the reduction of speed and acceleration during the light/dark cycle even in the light phase where 6-OHDA-lesioned rats were more active than control rats. These findings may indicate that 6-OHDA-lesioned animals started to present difficulty in the movement. Thus, the assumption of the scarcely motor symptoms as a consequence of circadian disruption reinforces our hypothesis about 6OHDA enables to induce mild motor symptoms in this model and although they are almost undetectable by conditional motor test. Early motor impairment might be detected by a sophisticated continuous monitoring system like the system we propose.

On the other hand, due to Shannon entropy is often considered as one of the classic and most natural way to measure the expected value (average) of the information in a dysfunctional signal (Eguiraun et al., 2018; Lópezde-Ipiña et al., 2019), the increase of entropy exhibited by 6-OHDA-lesioned rats confirmed the incipient sleep behavioral disorder.

Moreover, the reliability of this model was also confirmed by the high sensibility and specificity that we found allowing us the correct screening of both animal groups. Taken altogether, we propose the sleep disruption as a promising biomarker to be addressed using the 6-OHDA model in the reported conditions.

Concerning the motor evaluation developed with amphetamine test, the mild increased number of IL rotations was related to a mild degree of denervation in the dorsal subregion of the striatum and with the subsequent mild loss of dopaminergic terminals and neuronal cells in the SN. Interestingly, this correlation pointed out to the accurately or sensitivity of the amphetamine test predicting the dopamine decline (Decressac et al., 2012). Moreover, the mild motor deficit displayed by 6-OHDA-lesioned rats may result in the circadian locomotor activity reduction previously reported in the monitoring analysis. On the other hand, motor evaluation of 6-OHDA lesion was only carried out with the amphetamine test due to the apomorphine test, which is based on the subcutaneous administration of apomorphine to test CL rotations, only predicts an extensive lesion (nearly complete dopamine depletion) (Hudson et al., 1993; Dziewczapolski et al., 1996; Deumens et al., 2002; Yuan et al., 2005; Bilbao et al., 2006). In contrast, the amphetamine test detects motor impairments with only $50 \%$ neuronal loss allowing us to assess more moderate lesions (Bilbao et al., 2006). Therefore, the amphetamine test is commonly used in the 6-0HDA model to monitor motor changes and predict the degree of the lesion (Björklund and Dunnett, 2019).

The loss of dopaminergic neurons or fibers found in this model was around $45-55 \%$, indicating that the degree of lesion was lower than the results reported 3 weeks after lesion (Requejo et al., 2018a,b). Thus, the dopamine depletion did not reach the required threshold to develop motor symptoms (Deumens et al., 2002; Padmanabhan and Burke, 2018). However, this loss of dopamine could be sufficient to induce disruption of the circadian rhythms contrary to other studies which suggested a higher dopaminergic neuronal loss (about 60\%) in order to detect disruption of circadian locomotor (Souza et al., 2018). In addition, results indicated that a selective vulnerability to the toxin could be observed, pointing out to a dopamine decline topologically distributed in the $\mathrm{SN}$, in concordance with our previous works (Requejo et al., 2015, 2017). In 
fact, most of the dopaminergic neurons and dopaminergic terminals remained in the caudal axis, decreasing the survival toward rostral axis.

Regarding the mechanisms may underlay the early 6-OHDA-induced neurodegenerative effect on the dopaminergic system; we tested the effect of this toxin over apoptotic cascade and the survival signaling pathways in the striatum and SN. The toxicity mediated by 6 -OHDA was able to activate the proapoptotic pathway in neurons increasing caspase-3 levels in the striatum and SN. Indeed, the most remarkable outcomes were found in the striatum where the toxin was injected. These findings supported the capacity of 6-OHDA to trigger the activation of caspase-3 for inducing the cell death through the production of ROS leading to the apoptosis in agreement with other studies (Hernandez-Baltazar et al., 2013). Thus, the early reduction of ROS production could be a strategy to avoid proapoptotic events and the subsequent cell death.

Protein kinase B (AKT) signaling pathway as well as extracellular signal-regulated kinase (ERK) activation play a pivotal role in the regulation of cellular survival and in the inhibition of cell death (Smith and Dahodwala, 2014). However, the effect of 6-OHDA 2 weeks after administration was not sufficient to induce remarkable changes either in AKT or ERK expression in the nigrostriatal pathway. However, our previous studies have shown that the activation of both survival pathways were reduced significantly 3 weeks after 6-OHDA administration (Requejo et al., 2018b). Thereby, although proapoptotic events were evident 2 weeks after lesion, additional time will be required in order to detect changes in survival pathways.

Finally, for the present study the use of EE cages was only with the purpose of monitoring the behavior and assessing functional parameters. In fact, we propose a novel tracking system that allows monitoring animals in their home cages automatically detecting and categorizing behaviors without conditioning their regular environment. Interestingly, our observations indicated that monitored EE cages provided novel additional information for screening changes in the circadian function by monitoring parameters of circadian locomotor activity rhythms (activity area, speed and acceleration, among others) automatically during the light/dark cycle. Since there are discrepancies concerning to the parameters and methods for the evaluation of the active state of the animals (De Castro Medeiros et al., 2019), this monitoring system may represent a promising strategy offering a plethora of different activity parameters to assess along with other additional information about the state of the animal during the light/dark cycle.

\section{CONCLUSION}

In conclusion, we provide evidences about the suitability of the 6-OHDA-induced model reproducing the prodromal sleep disruption and we implemented a novel system to monitor behavior changes providing an optimal system to test different neuroprotective/neuroregenerative strategies.

\section{DATA AVAILABILITY STATEMENT}

The raw data supporting the conclusions of this article will be made available by the authors, without undue reservation, to any qualified researcher.

\section{ETHICS STATEMENT}

The animal study was reviewed and approved by the Ethical Committee and Animal Welfare of the University of the Basque Country Country (UPV/EHU, CEBA M20/2015/024).

\section{AUTHOR CONTRIBUTIONS}

CR designed the studio, performed the experiments, analyzed the results, prepared the figures, and wrote the manuscript. KL-d-I, EF, and PC analyzed the data from the monitoredEE cages and prepared Figures 1-3. JR-O, TM-H, and CM performed the stereotaxic lesions. LC-G contributed to the immunohistochemistry experiments. HC designed the monitored-EE cages. LU designed the studio, procured funding, provided laboratory resources, and reviewed the manuscript. JL conceived and designed the studio, supervised the work, procured funding, reviewed, and corrected the manuscript. All authors approved the final version of this manuscript.

\section{FUNDING}

This study has been financially supported by the University of the Basque Country (UPV/EHU) PPG 17/51 and GIU 092/19, the Basque Government (Saiotek SA-2010/00028, ELEKIN, Engineering and Society and Bioengineering, and ELKARTEK 18/99), "Ministerio de Ciencia e Innovación" (SAF2016 77758 R), FEDER funds, the European Union COST Action (CA15225, CA18106), DomusVi Foundation (FP18/76), and Government of Gipuzkoa (HELENA: Multisensory stimulation tools for Alzheimer Disease). CR appreciates the previous economic support received from UPV/EHU and the current postdoctoral fellowship received from Alfonso Martin Escudero Foundation.

\section{ACKNOWLEDGMENTS}

We thank SGIker (UPV/EHU) for their technical and human support. This manuscript has been released as a pre-print at Research Square (Requejo et al., 2020).

\section{SUPPLEMENTARY MATERIAL}

The Supplementary Material for this article can be found online at: https://www.frontiersin.org/articles/10.3389/fnins.2020. 590029/full\#supplementary-material 


\section{REFERENCES}

Ben, V., and Bruguerolle, B. (2000). Effects of bilateral striatal 6-OHDA lesions on circadian rhythms in the rat A radiotelemetric study. Life Sci. 67, 1549-1558. doi: 10.1016/S0024-3205(00)00751-757

Berg, D., Lang, A. E., Postuma, R. B., Maetzler, W., Deuschl, G., Gasser, T., et al. (2013). Changing the research criteria for the diagnosis of Parkinson's disease: obstacles and opportunities. Lancet Neurol. 12, 514-524. doi: 10.1016/S14744422(13)70047-70044

Bilbao, G., Ruiz-Ortega, J. A., Miguens, N., Ulibarri, I., Linazasoro, G., Gómez-Urquijo, S., et al. (2006). Electrophysiological characterization of substantia nigra dopaminergic neurons in partially lesioned rats: effects of subthalamotomy and levodopa treatment. Brain Res. 1084, 175-184. doi: 10. 1016/j.brainres.2006.02.052

Björklund, A., and Dunnett, S. B. (2019). The amphetamine induced rotation test: a re-assessment of its use as a tool to monitor motor impairment and functional recovery in rodent models of Parkinson's disease. J. Parkinson's Dis. 9, 17-29. doi: $10.3233 /$ JPD- 181525

Bradford, M. (1976). A rapid and sensitive method for the quantitation of microgram quantities of protein utilizing the principle of protein-dye binding. Anal. Biochem. 72, 248-254. doi: 10.1006/abio.1976.9999

Chiu, K., Lau, W. M., Lau, H. T., So, K. F., and Chang, R. C. C. (2007). Microdissection of rat brain for RNA or protein extraction from specific brain region. J. Visual. Exp. 269-269. doi: 10.3791/269

Ciric, J., Kapor, S., Perovic, M., and Saponjic, J. (2019). Alterations of sleep and sleep oscillations in the hemiparkinsonian rat. Front. Neurosci. 13:148. doi: 10.3389/fnins.2019.00148

De Castro Medeiros, D., Aguiar, C. L., Moraes, M. F. D., and Fisone, G. (2019). Sleep disorders in rodent models of Parkinson's disease. Front. Pharmacol. 10:1414. doi: 10.3389/fphar.2019.01414

De Lazzari, F., Bisaglia, M., Zordan, M. A., and Sandrelli, F. (2018). Circadian rhythm abnormalities in parkinson's disease from humans to flies and back. Int. J. Mol. Sci. 19:3911. doi: 10.3390/ijms19123911

Decressac, M., Mattsson, B., and Björklund, A. (2012). Comparison of the behavioural and histological characteristics of the 6-OHDA and $\alpha$-synuclein rat models of Parkinson 's disease. Exp. Neurol. 235, 306-315. doi: 10.1016/j. expneurol.2012.02.012

Deumens, R., Blokland, A., and Prickaerts, J. (2002). Modeling Parkinson's disease in rats: an evaluation of 6-OHDA lesions of the nigrostriatal pathway. Exp. Neurol. 175, 303-317. doi: 10.1006/exnr.2002.7891

Duty, S., and Jenner, P. (2011). Animal models of Parkinson's disease: a source of novel treatments and clues to the cause of the disease. Br. J. Pharmacol. 164, 1357-1391. doi: 10.1111/j.1476-5381.2011.01426.x

Dziewczapolski, G., Mora, M. A., Menalled, L. B., Stéfano, F. J. E., Rubinstein, M., and Gershanik, O. S. (1996). Threshold of dopamine content and D1 receptor stimulation necessary for the expression of rotational behavior induced by D2 receptor stimulation under normo and supersensitive conditions. NaunynSchmiedeberg's Arch. Pharmacol. 355, 30-35. doi: 10.1007/PL00004914

Eguiraun, H., Casquero, O., Sørensen, A. J., and Martinez, I. (2018). Reducing the number of individuals to monitor shoaling fish systems - application of the Shannon entropy to construct a biological warning system model. Front. Physiol. 9:493. doi: 10.3389/fphys.2018.00493

Fawcett, T. (2006). An introduction to ROC analysis. Pattern Recogn. Lett. 27, 861-874. doi: 10.1016/j.patrec.2005.10.010

Fifel, K. (2017). Alterations of the circadian system in Parkinson's disease patients. Movem. Dis. 32, 682-692. doi: 10.1002/mds.26865

Grandi, L. C., Di Giovanni, G., and Galati, S. (2018). Animal models of earlystage Parkinson's disease and acute dopamine deficiency to study compensatory neurodegenerative mechanisms. J. Neurosci. Methods 308, 205-218. doi: 10. 1016/j.jneumeth.2018.08.012

Gravotta, L., Gavrila, A. M., Hood, S., and Amir, S. (2011). Global depletion of dopamine using intracerebroventricular 6-hydroxydopamine injection disrupts normal circadian wheel-running patterns and PERIOD2 expression in the rat forebrain. J. Mol. Neurosci. 45, 162-171. doi: 10.1007/s12031-011-9520-9528

Hanley, J. A., and McNeil, B. J. (1982). The meaning and use of the area under a receiver operating characteristic (ROC) curve. Radiology 143, 29-36. doi: 10.1148/radiology.143.1.7063747
Hernandez-Baltazar, D., Mendoza-Garrido, M. E., and Martinez-Fong, D. (2013). Activation of GSK-3?? and Caspase-3 occurs in nigral dopamine neurons during the development of apoptosis activated by a striatal injection of 6Hydroxydopamine. PLoS One 8:e70951. doi: 10.1371/journal.pone.0070951

Howard, V., and Reed, M. (2004). Unbiased Stereology: Three-Dimensional Measurement in Microscopy. Available at: https://books.google.com/books? $\mathrm{hl}=$ en\&lr=\&id=MqR5AgAAQBAJ\&oi=fnd\&pg=PP1\&ots=cjiw_Bqun\&sig= TiyvemLqtAyPB2W_DEDFKYgc [Accessed August 29, 2020].

Hudson, J. L., van Horne, C. G., Strömberg, I., Brock, S., Clayton, J., Masserano, J., et al. (1993). Correlation of apomorphine- and amphetamine-induced turning with nigrostriatal dopamine content in unilateral 6-hydroxydopamine lesioned rats. Brain Res. 626, 167-174. doi: 10.1016/0006-8993(93)90576-90579

Iarkov, A., Barreto, G. E., Grizzell, J. A., and Echeverria, V. (2020). Strategies for the treatment of Parkinson's disease: beyond dopamine. Front. Aging Neurosci. 12. doi: 10.3389/fnagi.2020.00004

Isobe, Y., and Nishino, H. (2001). Circadian rhythm of drinking and running-wheel activity in rats with 6-hydroxydopamine lesions of the ventral tegmental area. Brain Res. 899, 187-192. doi: 10.1016/S0006-8993(01)02223-2225

Kirik, D., Rosenblad, C., and Björklund, A. (2000). Preservation of a functional nigrostriatal dopamine pathway by GDNF in the intrastriatal 6-OHDA lesion model depends on the site of administration of the trophic factor. Eur. J. Neurosci. 12, 3871-3882.

Ko, W. K. D., and Bezard, E. (2017). Experimental animal models of Parkinson's disease: a transition from assessing symptomatology to $\alpha$-synuclein targeted disease modification. Exp. Neurol. 298, 172-179. doi: 10.1016/j.expneurol.2017. 07.020

Lafuente, J. V., Requejo, C., Carrasco, A., and Bengoetxea, H. (2017). Nanoformulation: a useful therapeutic strategy for improving neuroprotection and the neurorestorative potential in experimental models of Parkinson's disease. Int. Rev. Neurobiol. 137, 99-122. doi: 10.1016/bs.irn.2017.09.003

Liepelt-Scarfone, I., Brändle, B., Yilmaz, R., Gauss, K., Schaeffer, E., Timmers, M., et al. (2017). Progression of prodromal motor and non-motor symptoms in the premotor phase study - 2-year follow-up data. Eur. J. Neurol. 24, 1369-1374. doi: 10.1111/ene.13397

López-de-Ipiña, K., Cepeda, H., Requejo, C., Fernandez, E., Calvo, P., and Lafuente, J. (2019). “Machine learning methods for environmental-enrichmentrelated variations in behavioral responses of laboratory rats.international work-conference on the interplay between natural and artificial computation, IWINAC 2019," in Understanding the Brain Function and Emotions (Springer), eds J. Toledo Moreo, and H. Adeli, (Cham: Springer), 420-427. doi: 10.1007/ 978-3-030-19591-5

Mahlknecht, P., Seppi, K., and Poewe, W. (2015). The concept of prodromal Parkinson's disease. J. Parkinson's Dis. 5, 681-697. doi: 10.3233/JPD-150685

Manchanda, S., and Sharma, S. (2016). "Analysis of computer vision based techniques for motion detection," in Proceedings of the 2016 6th International Conference - Cloud System and Big Data Engineering, Confluence 2016, (Piscataway, NJ: Institute of Electrical and Electronics Engineers Inc), 445-450. doi: 10.1109/CONFLUENCE.2016.7508161

Mantri, S., Morley, J. F., and Siderowf, A. D. (2018). The importance of preclinical diagnostics in Parkinson disease. Parkinsonism Related Dis. 64, 20-28. doi: 10.1016/j.parkreldis.2018.09.011

Masini, D., Lopes-Aguiar, C., Bonito-Oliva, A., Papadia, D., Andersson, R., Fisahn, A., et al. (2017). The histamine H3 receptor antagonist thioperamide rescues circadian rhythm and memory function in experimental parkinsonism. Trans. Psychiatry 7:e1088. doi: 10.1038/tp.2017.58

Miguelez, C., Aristieta, A., Cenci, M. A., and Ugedo, L. (2011). The locus coeruleus is directly implicated in L-DOPA-induced dyskinesia in parkinsonian rats: an electrophysiological and behavioural study. PLoS One 6:e24679. doi: 10.1371/ journal.pone.0024679

Miller, D. B., and O'Callaghan, J. P. (2015). Biomarkers of Parkinson's disease: present and future. Metabolism 64, S40-S46. doi: 10.1016/j.metabol.2014. 10.030

Padmanabhan, S., and Burke, R. E. (2018). Induction of axon growth in the adult brain: a new approach to restoration in Parkinson's disease. Movement Dis. 33, 62-70. doi: 10.1002/mds. 27209

Paxinos, G., and Watson, C. (2013). The Rat Brain in Stereotaxic Coordinates: Hard Cover Edition. Cambridge, MA: Academic Press. 
Rees, R. N., Noyce, A. J., and Schrag, A. (2019). The prodromes of Parkinson's disease. Eur. J. Neurosci. 49, 320-327. doi: 10.1111/ejn.14269

Requejo, C., Lopez, de Ipina, K., Fernández, E., Ruiz-Ortega, J. A., Calvo, P. M., et al. (2020). Changes in day/night Activity in the 6-OHDA-Induced Experimental Model of Parkinson's Disease: Exploring Prodromal Biomarkers. Available at https://www.researchsquare.com/article/rs-50664/v2 [Accessed August 4, 2020]

Requejo, C., Ruiz-Ortega, J. A., Bengoetxea, H., Bulnes, S., Ugedo, L., and Lafuente, J. V. (2018a). Deleterious effects of VEGFR2 and RET inhibition in a preclinical model of Parkinson's disease. Mol. Neurobiol. 55, 201-212. doi: 10.1007/s12035017-0733-x

Requejo, C., Ruiz-Ortega, J. A., Cepeda, H., Sharma, A., Sharma, H. S., Ozkizilcik, A., et al. (2018b). Nanodelivery of cerebrolysin and rearing in enriched environment induce neuroprotective effects in a preclinical rat model of Parkinson's disease. Mol. Neurobiol. 55, 286-299. doi: 10.1007/s12035-0170741-x

Requejo, C., Ruiz-Ortega, J. A., Bengoetxea, H., Garcia-Blanco, A., Herrán, E., Aristieta, A., et al. (2015). Topographical distribution of morphological changes in a partial model of Parkinson's disease-effects of nanoencapsulated neurotrophic factors administration. Mol. Neurobiol. 52, 846-858. doi: 10.1007/ s12035-015-9234-y

Requejo, C., Ruiz-Ortega, J. A., Bengoetxea, H., García-Blanco, A., Herrán, E., Aristieta, A., et al. (2017). Morphological changes in a severe model of Parkinson's disease and its suitability to test the therapeutic effects of microencapsulated neurotrophic factors. Mol. Neurobiol. 54, 7722-7735. doi: 10.1007/s12035-016-0244-241

Sakata, M., Sei, H., Toida, K., Fujihara, H., Urushihara, R., and Morita, Y. (2002). Mesolimbic dopaminergic system is involved in diurnal blood pressure regulation. Brain Res. 928, 194-201. doi: 10.1016/S0006-8993(01)03402-3403

Schapira, A. H. V., Chaudhuri, K. R., and Jenner, P. (2017). Non-motor features of Parkinson disease. Nat. Rev. Neurosci. 18, 435-450. doi: 10.1038/nrn.20 17.62

Schapira, A. H. V., and Tolosa, E. (2010). Molecular and clinical prodrome of Parkinson disease: implications for treatment. Nat. Rev. Neurol. 6, 309-317. doi: 10.1038/nrneurol.2010.52

Sengar, S. S., and Mukhopadhyay, S. (2016). "A novel method for moving object detection based on block based frame differencing," in Proceedinds of the 2016 $3 r d$ International Conference on Recent Advances in Information Technology, RAIT 2016, (Piscataway, NJ: Institute of Electrical and Electronics Engineers Inc), 467-472. doi: 10.1109/RAIT.2016.7507946

Sherbaf, F. G., Abadi, Y. R., Zadeh, M. M., Ashraf-Ganjouei, A., Moghaddam, H. S., and Aarabi, M. H. (2018). Microstructural changes in patients with parkinson's disease comorbid with REM sleep behaviour disorder and depressive symptoms. Front. Neurol. 9:441. doi: 10.3389/fneur.2018. 00441

Smith, K. M., and Dahodwala, N. (2014). Sex differences in Parkinson's disease and other movement disorders. Exp. Neurol. 259, 44-56. doi: 10.1016/J. EXPNEUROL.2014.03.010

Souza, L. C., Martynhak, B. J., Bassani, T. B., Turnes, J., de, M., Machado, M. M., et al. (2018). Agomelatine's effect on circadian locomotor rhythm alteration and depressive-like behavior in 6-OHDA lesioned rats. Physiol. Behav. 188, 298-310. doi: 10.1016/j.physbeh.2018.02.033

Tanaka, M., Yamaguchi, E., Takahashi, M., Hashimura, K., Shibata, T., Nakamura, W., et al. (2012). Effects of age-related dopaminergic neuron loss in the substantia nigra on the circadian rhythms of locomotor activity in mice. Neurosci. Res. 74, 210-215. doi: 10.1016/j.neures.2012.09.005

Weintraub, D., Tröster, A. I., Marras, C., and Stebbins, G. (2018). Initial cognitive changes in Parkinson's disease. Movem. Dis. 33, 511-519. doi: 10.1002/mds. 27330

West, M. J., Slomianka, L., and Gundersen, H. J. G. (1991). Unbiased stereological estimation of the total number of neurons in the subdivisions of the rat hippocampus using the optical fractionator. Anatom. Rec. 231, 482-497. doi: 10.1002/ar.1092310411

Willison, L. D., Kudo, T., Loh, D. H., Kuljis, D., and Colwell, C. S. (2013). Circadian dysfunction may be a key component of the non-motor symptoms of Parkinson's disease: insights from a transgenic mouse model. Exp. Neurol. 243, 57-66. doi: 10.1016/j.expneurol.2013.01.014

Yuan, H., Sarre, S., Ebinger, G., and Michotte, Y. (2005). Histological, behavioural and neurochemical evaluation of medial forebrain bundle and striatal 6-OHDA lesions as rat models of Parkinson's disease. J. Neurosci. Methods 144, 35-45. doi: 10.1016/j.jneumeth.2004.10.004

Conflict of Interest: The authors declare that the research was conducted in the absence of any commercial or financial relationships that could be construed as a potential conflict of interest.

Copyright $(2020$ Requejo, López-de-Ipiña, Ruiz-Ortega, Fernández, Calvo, MoreraHerreras, Miguelez, Cardona-Grifoll, Cepeda, Ugedo and Lafuente. This is an open-access article distributed under the terms of the Creative Commons Attribution License (CC BY). The use, distribution or reproduction in other forums is permitted, provided the original author $(s)$ and the copyright owner(s) are credited and that the original publication in this journal is cited, in accordance with accepted academic practice. No use, distribution or reproduction is permitted which does not comply with these terms. 